PROCEEDINGS OF THE

AMERICAN MATHEMATICAL SOCIETY

Volume 128, Number 5, Pages 1325-1332

S 0002-9939(99)05393-9

Article electronically published on October 5, 1999

\title{
A BOUND ON THE REDUCTION NUMBER OF A PRIMARY IDEAL
}

\author{
M. E. ROSSI
}

(Communicated by Wolmer V. Vasconcelos)

\begin{abstract}
Let $(A, \mathcal{M})$ be a local ring of positive dimension $d$ and let $I$ be an $\mathcal{M}$-primary ideal. We denote the reduction number of $I$ by $r(I)$, which is the smallest integer $r$ such that $I^{r+1}=J I^{r}$ for some reduction $J$ of $I$. In this paper we give an upper bound on $r(I)$ in terms of numerical invariants which are related with the Hilbert coefficients of $I$ when $A$ is Cohen-Macaulay. If $d=1$, it is known that $r(I) \leq e(I)-1$ where $e(I)$ denotes the multiplicity of $I$. If $d \leq 2$, in Corollary 1.5 we prove $r(I) \leq e_{1}(I)-e(I)+\lambda(A / I)+1$ where $e_{1}(I)$ is the first Hilbert coefficient of $I$. From this bound several results follow.

Theorem 1.3 gives an upper bound on $r(I)$ in a more general setting.
\end{abstract}

\section{INTRODUCTION}

Let $(A, \mathcal{M})$ be a local ring of dimension $d$ and let $I$ be an $\mathcal{M}$-primary ideal. If $J$ is a reduction of $I$, we will denote by $r_{J}(I)$ the smallest integer $r$ such that $I^{r+1}=J I^{r}$ and it will be called the reduction number of $I$ with respect to $J$.

The reductions of $I$ are ordered by inclusion with the smallest ones referred to as minimal reductions. The smallest reduction number attained among all minimal reductions is called the reduction number of $I$ and it will be noted by $r(I)$. If $(A, \mathcal{M})$ is a local ring with infinite residue field, then every $\mathcal{M}$-primary ideal $I$ has a minimal reduction and it is minimally generated by $d$ elements.

Let $A$ be a one-dimensional Cohen-Macaulay local ring. We denote by $(x)$ a minimal reduction of $I$ and by $H_{I}(n):=\lambda\left(I^{n} / I^{n+1}\right)$ the Hilbert function of $I$ where $\lambda()$ is the length as $A$-module. Then

$$
H_{I}(n)=e(I)-\lambda\left(I^{n+1} / x I^{n}\right)
$$

where $e(I)$ is the multiplicity of $I$.

From this equality it is clear that in this case the reduction number does not depend on $x$. More generally if $A$ is a $d$-dimensional Cohen-Macaulay local ring and $J$ is a minimal reduction of $I$, we denote by $G:=g r_{I}(A)=\bigoplus_{n \geq 0} I^{n} / I^{n+1}$ the associated graded ring of $I$. It is well known that if depth $G \geq d-1$, then $r_{J}(I)$ does not depend on the minimal reduction $J$.

Received by the editors July 6, 1998.

1991 Mathematics Subject Classification. Primary 14M05; Secondary $13 \mathrm{H} 10$.

Key words and phrases. Cohen-Macaulay local ring, primary ideals, reduction number, minimal reduction, associated graded ring, Hilbert function, Hilbert coefficients. 
Further, if $A$ is a one-dimensional Cohen-Macaulay local ring, by a generalization of Macaulay's theorem (see [B, Corollary 2.11]), we deduce

$$
H_{I}(n)=e(I)-\lambda\left(I^{n+1} / x I^{n}\right) \geq \min \{e(I), n+1\} .
$$

From this we get $I^{e(I)}=x I^{e(I)-1}$ and then $r(I) \leq e(I)-1$.

This bound can be generalized. For the reduction number of the maximal ideal $\mathcal{M}$ of a local Cohen-Macaulay ring $A$ of dimension $d>0$, J. Sally [S1] proved that

$$
r(\mathcal{M}) \leq d ! e(A)-1 .
$$

W. Vasconcelos ([VW, Remark 6.16]) improved this bound and, for any $\mathcal{M}$-primary ideal $I$, he proved

$$
r(I) \leq d e(I)-2 d+1 .
$$

This bound is the best known estimate for the reduction number of $I$.

In this paper we prove a different bound for $r(I)$ which involves different numerical invariants of $I$ (see Theorem 1.3). The new formula is an improvement for special ideals. For example, we consider a Cohen-Macaulay local ring $A$ and let $e_{1}(I)$ be the first coefficient of the Hilbert polynomial of $I$. If $e_{1}(I)$ is the smallest possible, that is, $e_{1}(I)=e(I)-\lambda(A / I)$, then $r(I)=1$ (see $[\mathrm{H}]$ and $[\mathrm{O}]$ ). In this case $r(I)$ is in general far from the integer $d e(I)-2 d+1$. Corollary 1.9 shows that this result and others can be obtained as consequences of the new bound.

In other cases, in order to prove good properties of the associated graded ring $G$ of $I$, we need to control the reduction number of $I$. For example, we recall that, by $[\mathrm{VV}]$, if $r_{J}(\mathcal{M}) \leq 2$ for some minimal reduction $J$ of $\mathcal{M}$, then the associated graded $\operatorname{ring} G$ of $\mathcal{M}$ is Cohen-Macaulay. Furthermore, Sally in [S2] stated that if we denote by $v$ the embedding dimension of $A$ and $e(A)=v-d+2$, then depth $G \geq d-1$. We recall that, in the same paper, she reduced the problem to prove a condition on the reduction number of $\mathcal{M}$. Several papers ([RV], [W], [E], [CPV] and $[R])$ deal with this problem. Essentially the proof of our main result comes from a deeper investigation of the methods developed in $[R V]$ and $[R]$.

\section{The BOUnd ON THE REDUCTION NUMBER}

Let $(A, \mathcal{M})$ be a local ring of positive dimension $d$ and let $I$ be an $\mathcal{M}$-primary ideal. We recall some general facts.

For every $n$ we consider the chain of ideals

$$
I^{n} \subseteq I^{n+1}: I \subseteq I^{n+2}: I^{2} \subseteq \cdots \subseteq I^{n+k}: I^{k} \subseteq \cdots
$$

This chain stabilizes at an ideal which was denoted by Ratliff and Rush as

$$
\widetilde{I^{n}}:=\bigcup_{k \geq 1}\left(I^{n+k}: I^{k}\right)
$$

If depth $A$ is positive, then $\widetilde{I^{n}}=I^{n}$ for $n>>0$. In particular $\widetilde{I^{n+1}} / J \widetilde{I^{n}}$ is a finite $A$-module for any minimal reduction $J$ of $I$. We will denote by $\lambda$ the length function on $A$-modules. We define for every $n \geq 0$

$$
\rho_{n}:=\lambda\left(\widetilde{I^{n+1}} / J \widetilde{I^{n}}\right) \text {. }
$$

These invariants come from the homological properties of $I$ and, if $A$ is CohenMacaulay, they can be related to the Hilbert coefficients of $I$ (see [HM, Section 
4]). It will be useful in the following to recall that, if $A$ is Cohen-Macaulay and $\operatorname{dim} A \leq 2$, then

$$
e_{1}(I)=\sum_{n \geq 0} \rho_{n}
$$

Let $J$ be a minimal reduction of $I$; for every $n \geq 0$, we denote by $v_{n}$ the integers

$$
v_{n}:=\lambda\left(I^{n+1} / J I^{n}\right) \text {. }
$$

We recall that, if $A$ is a one-dimensional Cohen-Macaulay local ring, we have $H_{I}(n)=e(I)-v_{n}$ and in particular

$$
e_{1}(I)=\sum_{n=0}^{s-1} v_{n}
$$

where $s$ is the reduction number of $I$.

Lemma 1.1. Let $(A, \mathcal{M})$ be a local ring of positive depth and let $I$ be an $\mathcal{M}$ primary ideal. If $J$ is a minimal reduction of $I$ and $I^{n+1} \cap J=J I^{n}$ for some positive integer $n$, then

$$
\rho_{n}-v_{n}=\lambda\left(\widetilde{I^{n+1}} / J \widetilde{I^{n}}+I^{n+1}\right)
$$

Proof. We have

$$
\widetilde{I^{n}} \subseteq J \widetilde{I^{n}}+I^{n+1} \subseteq \widetilde{I^{n+1}}
$$

hence

$$
\lambda\left(\widetilde{I^{n+1}} / J \widetilde{I^{n}}+I^{n+1}\right)=\rho_{n}-\lambda\left(J \widetilde{I^{n}}+I^{n+1} / \widetilde{I^{n}}\right)=\rho_{n}-\lambda\left(I^{n+1} / J \widetilde{I^{n}} \cap I^{n+1}\right) .
$$

Since

$$
J I^{n} \subseteq J \widetilde{I^{n}} \cap I^{n+1} \subseteq I^{n+1} \cap J=J I^{n},
$$

we get $J I^{n}=\widetilde{I^{n}} \cap I^{n+1}$ and the conclusion follows.

The next result is a generalization of [RV, Proposition 2.4] and of [E, Proposition 2.5]. This will be a crucial point in our main result, so we include a proof, even though it is close to the proofs of the above-mentioned propositions.

Let $\mathcal{R}(I):=\bigoplus_{n \geq 0} I^{n}=A[I T]$ be the Rees algebra of $I$ and let $M$ be a graded $\mathcal{R}(I)$-module. For every $n \geq 0$, we define

$$
\operatorname{Ann}_{I^{n}}(M):=\left\{x \in I^{n}: x T^{n} M=0\right\} .
$$

If $J$ is an ideal of $A$ such that $J \subseteq I$, then we may view $M$ as a graded $\mathcal{R}(J)=$ $A[J T]$-module. On this module $\mathcal{R}(I)$ acts as endomorphisms over $\mathcal{R}(J)$. We will denote by $\mathcal{R}(J)_{+}$the ideal $\bigoplus_{n>0} J^{n}$.

Note the following isomorphism of $\mathcal{R}(J)$-modules which will be important in our arguments.

For every $n \geq 0$ we have

$$
\left(M / \mathcal{R}(J)_{+} M\right)_{n} \simeq M_{n} /\left(J^{n} M_{0}+J^{n-1} M_{1}+\cdots+J M_{n-1}\right) .
$$

Proposition 1.2. Let $I$ and $J$ be ideals of a local ring $A$ with $J \subseteq I$ and let $M$ be an $\mathcal{R}(I)$-module of finite length as A-module. Let $\nu$ be the minimal number of generators of $M / \mathcal{R}(J)_{+} M$ as $A$-module. Then

$$
I^{\nu}=J I^{\nu-1}+A n n_{I^{\nu}}(M) .
$$


Proof. Let $p$ be the largest integer such that $M_{p} \neq 0$. For all $n=0, \ldots, p$ we consider the elements $m_{1 n}, \ldots, m_{\nu_{n} n} \in M_{n}$ such that the corresponding elements in $\left(M / \mathcal{R}(J)_{+} M\right)_{n}$ form a minimal system of generators as $A$-module. We have $\nu=\sum_{n=0}^{p} \nu_{n}$ and $|(i n)|=\nu$ if $n=0, \ldots, p$ and $i=1, \ldots, \nu_{n}$.

If $a_{i n}$ is an element of $I$, since

$$
\left(M / \mathcal{R}(J)_{+} M\right)_{n+1} \simeq M_{n+1} /\left(J^{n+1} M_{0}+J^{n} M_{1}+\cdots+J M_{n}\right),
$$

there exist $c_{(i n)(k j)} \in J^{n+1-j}$ such that

$$
\left(a_{i n} T\right) m_{i n}=\sum_{j=0}^{n+1} \sum_{k=1}^{\nu_{j}} c_{(i n)(k j)} T^{n+1-j} m_{k j}
$$

with $m_{k} p+1=0$ for every $k$.

Thus if we consider the relations

$$
\sum_{j=0}^{n+1} \sum_{k=1}^{\nu_{j}} c_{(i n)(k j)} T^{n+1-j} m_{k j}-\left(a_{i n} T\right) m_{i n}=0
$$

we get a system of $\nu$ linear equations in the $\nu$ variables $m_{k j}$ where $j=0, \ldots, p$ and $k=1, \ldots, \nu_{j}$. The corresponding matrix $C$ has size $\nu \times \nu$ and entries which are homogeneous elements in the Rees ring $\mathcal{R}(I)$. Since the $(i n)(k j)$-entry has degree $n+1-j$ if $n+1 \geq j$ and is zero otherwise, we may assign degree $n+1-j$ to the $(i n)(k j)$-entry of $C$ whatsoever. This implies that every two by two minor of $C$ is a homogenous element; hence its determinant $\operatorname{det}(C)$ is homogeneous too and its degree is $\nu$ because the elements on the diagonal $(i n)=(k j)$, which are $\left(c_{(i n)(i n)}-a_{i n}\right) T$, all have degree 1.

If $a=\prod a_{i n}$ for $n=0, \ldots, p$ and $i=1, \ldots, \nu_{n}$, it is easy to see that

$$
\operatorname{det}(C)=(-1)^{\nu}(a-\sigma) T^{\nu}
$$

for a suitable $\sigma \in J I^{\nu-1}$. Since by the Cayley-Hamilton theorem, $\operatorname{det}(C)$ kills all the variables $m_{i n}$, for $n=0, \ldots, p$ and $i=1, \ldots, \nu_{n}$, we get

$$
(a-\sigma) T^{\nu} M=0
$$

and hence $a-\sigma \in A n n_{I^{\nu}}(M)$.

We may repeat the same procedure for all monomial $a=\prod a_{i n}$ in $I^{\nu}$ and the result follows.

In the following, if $J$ is a minimal reduction of $I$, we denote

$$
S_{J}:=\left\{n \in \mathbb{N} / I^{j+1} \cap J=J I^{j} \text { for all } j \leq n\right\} .
$$

Observe that $0 \in S_{J}$ and, if $I$ is integrally closed, then $1 \in S_{J}$.

Theorem 1.3. Let $(A, \mathcal{M})$ be a local ring of positive depth and let $I$ be an $\mathcal{M}$ primary ideal. If $J$ is a minimal reduction of $I$ and $n \in S_{J}$, then

$$
r_{J}(I) \leq \sum_{i \geq 0} \rho_{i}+n+1-\sum_{i=0}^{n} v_{i}
$$

Proof. We denote by $M$ the $\mathcal{R}(I)$-graded module $M:=\bigoplus_{i \geq 1} \widetilde{I^{i}} / I^{i}$, so that in particular $M$ is a finite $A$-module. We recall that for every $j \geq 0$ we have

$$
\left(M / \mathcal{R}(J)_{+} M\right)_{j+1} \simeq M_{j+1} /\left(J^{j+1} M_{0}+J^{j} M_{1}+\cdots+J M_{j}\right)
$$


and it is easy to see that

$$
M_{j+1} /\left(J^{j+1} M_{0}+J^{j} M_{1}+\cdots+J M_{j}\right) \simeq \widetilde{I^{j+1}} / J \widetilde{I^{j}}+I^{j+1} .
$$

We have $\lambda\left(\widetilde{I^{j+1}} / J \widetilde{I^{j}}+I^{j+1}\right) \leq \rho_{j}=\lambda\left(\widetilde{I^{j+1}} / J \widetilde{I^{j}}\right)$ and the equality holds if and only if $I^{j+1} \subseteq J \widetilde{I^{j}}$. Let $k$ be the least integer $j$ such that $I^{j+1} \subseteq J \widetilde{I}^{j}$.

Let $\nu_{j}$ be the minimal number of generators of $\widetilde{I^{j+1}} / J \widetilde{I}^{j}+I^{j+1}$ as $A$-module. Clearly $\nu_{j} \leq \lambda\left(\widetilde{I^{j+1}} / J \widetilde{I^{j}}+I^{j+1}\right)$ for every $j$. If $\nu=\sum_{j \geq 0} \nu_{j}$, by Proposition 1.2, we get

$$
I^{\nu}=J I^{\nu-1}+A n n_{I^{\nu}}(M)
$$

and therefore

$$
\begin{gathered}
I^{\nu+k+1}=I^{\nu} I^{k+1}=I^{k+1}\left(J I^{\nu-1}+A n n_{I^{\nu}}(M)\right) \\
=J I^{\nu+k}+I^{k+1} A n n_{I^{\nu}}(M) \subseteq J I^{\nu+k}+\widetilde{I^{k}} A n n_{I^{\nu}}(M) \subseteq J I^{\nu+k} .
\end{gathered}
$$

It follows that

$$
r_{J}(I) \leq \nu+k=\sum_{j \geq 0} \nu_{j}+k \leq \sum_{j \geq 0} \lambda\left(\widetilde{I^{j+1}} / J \widetilde{I^{j}}+I^{j+1}\right)+k .
$$

Now if $n \in S_{J}$, then by Lemma $1.1, \rho_{j}=v_{j}+\lambda\left(\widetilde{I^{j+1}} / J \widetilde{I^{j}}+I^{j+1}\right)$ for every $j \leq n$. In particular

$$
r_{J}(I) \leq \sum_{j=0}^{n}\left(\rho_{j}-v_{j}\right)+\sum_{j \geq n+1} \lambda\left(\widetilde{I^{j+1}} / J \widetilde{I^{j}}+I^{j+1}\right)+k .
$$

If $k \leq n+1$, the result follows. We may suppose $k \geq n+2$; then by the true definition of $k$, we have

$$
\begin{gathered}
r_{J}(I) \leq \sum_{j=0}^{n}\left(\rho_{j}-v_{j}\right)+\sum_{j=n+1}^{k-1}\left(\rho_{j}-1\right)+\sum_{j \geq k} \rho_{j}+k \\
=\sum_{j=0}^{n}\left(\rho_{j}-v_{j}\right)+\sum_{j \geq n+1} \rho_{j}-(k-1-n)+k=\sum_{j \geq 0} \rho_{j}+n+1-\sum_{j=0}^{n} v_{j} .
\end{gathered}
$$

Remark 1.4. If depth $G>0$ or equivalently $\widetilde{I}^{j}=I^{j}$ for all $j$, by Theorem 1.3 we obtain

$$
r_{J}(I) \leq n+1+\sum_{i \geq n+1} v_{i}
$$

for any minimal reduction $J$ of $I$ and $n \in S_{J}$.

If $(A, \mathcal{M})$ is a Cohen-Macaulay local ring of dimension $d \leq 2$, then $e_{1}(I)=$ $\sum_{j>0} \rho_{j}($ see $[\mathrm{HM}])$. Since $0 \in S_{J}$ and $v_{0}=\lambda(I / J)=e(I)-\lambda(A / I)$, by Theorem 1.3 we obtain the following result.

Corollary 1.5. Let $(A, \mathcal{M})$ be a Cohen-Macaulay local ring of dimension $d \leq 2, I$ an $\mathcal{M}$-primary ideal and $J$ a minimal reduction of $I$. Then

$$
r_{J}(I) \leq e_{1}(I)-e(I)+\lambda(A / I)+1
$$

The following example shows that the maximum value can be reached. 
Example 1.6. We consider $A=k[\mid X, Y, Z \|] /\left(Z^{3}\right)$ and the ideal $I=\left(x^{2}, y^{2}, x z, y z\right)$ in $A$. Then $J=\left(x^{2}, y^{2}\right)$ is a minimal reduction of $I$. We have $e_{1}(I)=8, e(I)=12$, $\lambda(A / I)=6$ and $r_{J}(I)=3$.

Let $\mathcal{F}$ be the Ratliff-Rush filtration and we consider $G(\mathcal{F})=\bigoplus_{n \geq 0} \widetilde{I^{n}} / \widetilde{I^{n+1}}$. We remark that Corollary 1.5 holds under the weaker assumption depth $G(\mathcal{F}) \geq d-1$. In fact, if depth $G(\mathcal{F}) \geq d-1$, then by [HM, Proposition 4.6], we have $e_{1}(I)=$ $\sum_{j \geq 0} \rho_{j}$ and we can get the same conclusion.

The next results show that in some case it is possible to control the depth of the associated graded ring $G$ by using information on the reduction number of $I$.

From Corollary 1.5 we obtain a proof of a well known conjecture stated by Sally in [S2]. The conjecture was proved in $[\mathrm{RV}]$ and $[\mathrm{W}]$ in the case of the maximal ideal and in $[\mathrm{CPV}],[\mathrm{E}],[\mathrm{R}]$ for any $\mathcal{M}$-primary ideal. We now give a short proof of this fact by using the results of this paper. More details concerning the first part of the proof can be found in [S2], [RV] and [R].

We recall that an element $x$ in $I$ is called superficial for $I$ if there exists an integer $c>0$ such that

$$
\left(I^{n}: x\right) \cap I^{c}=I^{n-1}
$$

for every $n>c$. If the residue field is infinite, superficial elements always exist and if depth $A$ is positive, every superficial element for $I$ is also a regular element in $A$. By passing, if needed, to the local ring $A[X]_{\mathcal{M} A[X]}$ we may assume that the residue field is infinite.

A sequence $x_{1}, \ldots, x_{r}$ in the local ring $(A, \mathcal{M})$ is called a superficial sequence for $I$, if $x_{1}$ is superficial for $I$ and $\overline{x_{i}}$ is superficial for $I /\left(x_{1}, \ldots, x_{i-1}\right)$ for $2 \leq i \leq r$.

If $A$ is a local ring of dimension $d>0$, then we can find a maximal superficial sequence $x_{1}, \ldots, x_{d}$ for $I$.

If $J=\left(x_{1}, \ldots, x_{r}\right)$, then the following equality on the Hilbert coefficients holds:

$$
e_{i}(A)=e_{i}(A / J)
$$

for every $i=0, \ldots, d-r$.

Moreover there is a very important trick (the so-called Sally machine) to reduce dimension in question relating to depth properties of the associated graded ring (see [HM, Lemma 2.2]). We have

$$
\operatorname{depthgr~}_{I}(A) \geq r+1 \Longleftrightarrow \operatorname{depthgr~}_{I / J}(A / J) \geq 1 .
$$

Remark that if $J$ is an ideal generated by a maximal superficial sequence in $I$, then $J$ is a minimal reduction of $I$. Conversely from [S, Theorem 4], if $J$ is a minimal reduction of $I$, then there exists a minimal system of generators of $J$ which is a maximal superficial sequence for $I$.

Corollary 1.7. Let $(A, \mathcal{M})$ be a Cohen-Macaulay local ring of dimension $d$ and $I$ an $\mathcal{M}$-primary ideal. If

$$
e(I)=\lambda\left(I / I^{2}\right)+(1-d) \lambda(A / I)+1,
$$

then depth $G \geq d-1$.

Proof. From [V], the assumption $e(I)=\lambda\left(I / I^{2}\right)+(1-d) \lambda(A / I)+1$ is equivalent to $\lambda\left(I^{2} / J I\right)=1$. Now, by using the Sally machine, we may reduce the problem to the case $d=2$. Let $x, y$ be a superficial sequence for $I$ and $J=(x, y)$. 
Since $\lambda\left(I^{2} / J I\right)=1$ we may write $I^{2}=J I+(a b)$ for some $a$ and $b$ in $I$. Then for every $n \geq 1$ we have a surjection from $I^{n+1} / J I^{n}$ to $I^{n+2} / J I^{n+1}$ by multiplication by $a$ and consequently $\lambda\left(I^{n+1} / J I^{n}\right) \leq 1$ for every $n \geq 1$.

We consider $\bar{I}=I /(x)$ and let $s$ be the reduction number of $\bar{I}$. We may suppose $s \geq 2$, otherwise again by the Sally machine we have $G$ Cohen-Macaulay. It is clear that $\lambda\left(\bar{I}^{j+1} / y \bar{I}^{j}\right)=1=\lambda\left(I^{j+1} / J I^{j}\right)$ for $j=1, \ldots, s-1$ and zero otherwise.

For every $j \geq 0$ there is an exact sequence

$$
0 \longrightarrow I^{j}: x / I^{j}: J \stackrel{y}{\longrightarrow} I^{j+1}: x / I^{j} \stackrel{x}{\longrightarrow} I^{j+1} / J I^{j} \longrightarrow \bar{I}^{j+1} / y \bar{I}^{j} \longrightarrow 0 .
$$

By induction on $j$ it is easy to see that if we prove $r_{J}(I) \leq s$, then $I^{j+1}: x=I^{j}$ for every $j$ and so depth $G>0$, as required. But $e_{1}(\bar{I})=\sum_{j \geq 0} \lambda\left(\bar{I}^{j+1} / y \bar{I}^{j}\right)=$ $e(I)-\lambda(A / I)+s-1$. Since $e_{1}(\bar{I})=e_{1}(I)$, the conclusion follows by Corollary 1.5 .

Remark 1.8. Let $(A, \mathcal{M})$ be a Cohen-Macaulay local ring of dimension $d \leq 2$ and $I$ an $\mathcal{M}$-primary ideal. If $J$ is a minimal reduction of $I$ such that $I^{2} \cap J=J I$, we may improve the bound obtained in Corollary 1.5. In this case $1 \in S_{J}$ and so, by Theorem 1.3, we have $r_{J}(I) \leq \sum_{i \geq 0} \rho_{i}+2-v_{0}-v_{1}$. Now $\sum_{j \geq 0} \rho_{j}=e_{1}(I)$, $v_{0}=e(I)-\lambda(A / I)$ and, by $[\mathrm{V}], v_{1}=\lambda\left(I^{2} / J I\right)=e(I)-\lambda\left(I / I^{2}\right)+\lambda(A / I)$. It follows that

$$
r_{J}(I) \leq e_{1}(I)+2-e(I)+\lambda(A / I)-\lambda\left(I^{2} / J I\right)=e_{1}(I)-2 e(I)+\lambda\left(I / I^{2}\right)+2 .
$$

We end by giving easy proofs of a collection of results proved in $[\mathrm{H}],[\mathrm{O}],[\mathrm{HM}]$, $[\mathrm{I}]$ and $[\mathrm{GR}]$ now using the techniques developed in this paper.

Corollary 1.9. Let $(A, \mathcal{M})$ be a Cohen-Macaulay local ring of dimension $d$ and $I$ an $\mathcal{M}$-primary ideal.

i) If $e_{1}(I)=e(I)-\lambda(A / I)$, then $r(I) \leq 1$ and $G$ is Cohen-Macaulay.

ii) If $e_{1}(I)=e(I)-\lambda(A / I)+1$ and $I^{2} \cap J=J I$ for some minimal reduction $J$ of $I$, then $r(I) \leq 2$ and $G$ is Cohen-Macaulay.

iii) If $e_{1}(I)=e(I)-\lambda(A / I)+2$ and $I^{2} \cap J=J I$ for some minimal reduction $J$ of $I$, then $r(I) \leq 3$ and depth $G \geq d-1$.

Proof. By using the Sally machine and the good behaviour of $e_{1}(I)$ modulo superficial elements, we may reduce the first two statements to dimension one and the last to dimension two.

If $e_{1}(I)=e(I)-\lambda(A / I)$, by Corollary 1.5 we have $r(I) \leq 1$. Then $I^{2}=J I$ and by [VV] it follows that $G$ is Cohen-Macaulay.

If $e_{1}(I)=e(I)-\lambda(A / I)+1$, by Corollary 1.5 we have $r(I) \leq 2$. Then $I^{3}=J I^{2}$ and $I^{2} \cap J=J I$ and again by [VV] it follows that $G$ is Cohen-Macaulay.

If $e_{1}(I)=e(I)-\lambda(A / I)+2$, by Corollary 1.5 we have $r(I) \leq 3$. If $r(I) \leq 2$, then $G$ is Cohen-Macaulay as before, otherwise $r(I)=3$. By Remark 1.8, we have $r(I)=3 \leq 4-\lambda\left(I^{2} / J I\right)$ and the result follows by Corollary 1.7 .

\section{ACKNowledgement}

The author thanks G. Valla for helpful discussions. 


\section{REFERENCES}

[B] C. Blancafort, Hilbert functions of graded algebras over Artinian rings, J. of Pure and Applied Algebra 125 (1998), 55-78. MR 98m:13023

[CPV] A. Corso, C. Polini, M. Vaz Pinto, Sally Modules and associated graded rings, Communications in Algebra 26 (8) (1998), 2689-2708. CMP 98:15

[E] J. Elias, On the depth of the tangent cone and the growth of the Hilbert function, to appear in Trans. Amer. Math. Soc. CMP 98:07

[GR] A. Guerrieri, M. E. Rossi, Hilbert coefficients for Hilbert filtrations, J. Algebra 199 (1998), 40-61. MR 98i: 13027

[HM] S. Huckaba, T. Marley, Hilbert coefficients and the depths of associated graded rings, J. London Math. Soc. 56 (1997), 64-76. MR 98i:13028

[H] C. Huneke, Hilbert functions and symbolic powers, Michigan Math. J. 34 (1987), 293-318. MR 89b: 13037

[I] S. Itoh, Hilbert coefficients of integrally closed ideals, J. Algebra 176 (1995), 638-652. MR 96g: 13019

[N] D. G. Northcott, A note on the coefficients of the abstract Hilbert function, J. London Math. Soc. 35 (1960), 209-214. MR 22:1599

[O] A. Ooishi, $\Delta$-genera and sectional genera of commutative rings, Hiroshima Math. J. 17 (1987), 361-372. MR 89f:13033

[RR] L. J. Ratliff, D. Rush, Two notes on reductions of ideals, Indiana Univ. Math. J. 27 (1978), 929-934. MR 58:22034

[R] M. E. Rossi, Primary ideals with good associated graded ring, to appear in J. of Pure and Applied Algebra.

[RV] M. E. Rossi, G. Valla, A conjecture of J. Sally, Communications in Algebra 24 (13) (1996), 4249-4261. MR 97j:13021

[S] I. Swanson, A note on the analytic spread, Communications in Algebra 22, 2 (1994), 407-411. MR 95b:13007

[S1] J. Sally, Bounds for numbers of generators for Cohen-Macaulay ideals, Pacific J. Math. 63 (1976), 517-520. MR 53:13208

[S2] J. Sally, Cohen-Macaulay local ring of embedding dimension $e+d-2$, J. Algebra 83 (1983), 325-333. MR 85c: 13017

[S3] J. Sally, Hilbert coefficients and reduction number 2, J. Algebraic Geom. 1 (1992), 325-533. MR 93b: 13026

[V] G. Valla, On form rings which are Cohen-Macaulay, J. Algebra 58 (1979), 247-250. MR 80h:13025

[VV] P. Valabrega, G. Valla, Form rings and regular sequences, Nagoya Math. J. 72(2) (1978), 475-481. MR 80d:14010

[VW] W. Vasconcelos, Cohomological Degrees of graded modules, Six Lectures on Commutative algebra, Progress in Math. 166 , Birkhauser, Boston (1998), 345-392. CMP 99:02

[W] H. Wang, On Cohen-Macaulay local rings with embedding dimension $e+d-2$, J. Algebra 190 (1997), 226-240. MR 98d:13027

Dipartimento di Matematica, Universita' di Genova, Via Dodecaneso 35, 16146- GenOVA, ITALY

E-mail address: rossim@dima.unige.it 